\title{
Preparation and thermal properties of mesoporous silica/phenolic resin nanocomposites via in situ polymerization
}

\author{
C. B. $Y u^{1,2}, C . W e i^{1 *}, J . L v^{1}, H . X . L i u^{1}$, L. T. Meng ${ }^{1}$ \\ ${ }^{1}$ Key Laboratory of New Processing Technology for Nonferrous Metals \& Materials Ministry of Education, and School of \\ Materials Science and Engineering, Guilin University of Technology, 541004 Guilin, China \\ ${ }^{2}$ College of Chemistry and Chemical Engineering, Guangxi University, 530004 Nanning, China
}

Received 21 February 2012; accepted in revised form 29 April 2012

\begin{abstract}
In order to enhance the adhesion between inorganic particles and polymer matrix, in this paper, the mesoporous silica SBA-15 material was synthesized by the sol-gel method. The surface of SBA-15 was modified using $\gamma$-glycidyloxypropyltrimethoxysilane (GOTMS) as a coupling agent, and then mesoporous silica/phenolic resin (SBA-15/PF) nanocomposites were prepared via in situ polymerization. The structural parameters and physical properties of SBA-15, SBA-15-GOTMS (SBA-15 surface treated using GOTMS as coupling agents) and E-SBA-15/PF (SBA-15/PF nanocomposites extracted using ethanol as solvent) were characterized by X-ray diffraction (XRD), $\mathrm{N}_{2}$ adsorption-desorption, Fourier transform infrared spectroscopy (FTIR), scanning electron microscopy (SEM), transmission electron microscopy (TEM) and thermogravimetric analysis (TGA). The thermal properties of the nanocomposites were studied by differential scanning calorimetry (DSC) and thermogravimetric analysis (TGA). The results demonstrated that the GOTMS were successfully grafted onto the surface of SBA-15, and chemical bonds between PF and SBA-15-GOTMS were formed after in situ polymerization. In addition, it is found that the in situ polymerization method has great effects on the textural parameters of SBA-15. The results also showed that the glass transition temperatures and thermal stability of the PF nanocomposites were obviously enhanced as compared with the pure PF at silica contents between $1-3 \mathrm{wt} \%$, due to the uniform dispersion of the modified SBA-15 in the matrix.
\end{abstract}

Keywords: thermal properties, $S B A-15$, phenolic resin

\section{Introduction}

Organic-inorganic hybrid materials, especially polymer matrix composites with inorganic nanoscale building blocks, have drawn the widespread attention of researchers owing to the promise of combining the superior mechanical and thermal properties of inorganic phases with the flexibility and processibility of organic polymers $[1,2]$. The comprehensive performances of the composites depended on many factors, such as the intrinsic properties of the polymers, the processing technology of the com- posites, the dispersion of the nanoparticles in the polymer matrix, and the interfacial compatibility between nanoparticles and the polymer matrix [3]. Commonly used inorganic nanoscale additives include carbon nanotubes, nanoparticles, layered silicates and clays. Mesoporous molecular sieve, a new class of nanoscale materials which possess large specific surface areas and tunable pore sizes between 2 and $50 \mathrm{~nm}$, have provided the possibility of incorporating diverse organic guest species into ordered mesoporous structures [4-5]. Although mesoporous molecular sieve have been widely stud-

\footnotetext{
${ }^{*}$ Corresponding author, e-mail: 1005668130@qq.com
}

(c) BME-PT 
ied as catalysts, absorbents, chemical sensing, and electronic device materials, using mesoporous molecular sieve as polymer additive attracted less attention [6-8]. Recently, mesoporous silica reinforced polymer composites have attracted considerable interests due to the possibility of improving the mechanical and thermal properties of polymers [917], and have even generated certain properties, for example, low dielectric constant [10, 12-14, 16, 17], low thermal expansion property [18-21], excellent friction and wear performance [22], and so on. It is a key factor that the incorporation of guest-monomer or polymer into the channels of mesoporous materials contributed to the dispersion of the additives in polymer matrix and the advancement of the properties of as-prepared composites. Run et al. [3] reported the preparation of MCM-48/PMMA composites by injecting MMA monomers into the mesoporous particles under high temperature and low pressure. They found that the physical properties and thermal stability of MCM-48/PMMA composites were improved significantly with the increase of the content of MCM-48.

Phenolic resins (PF), classified as resol and novolac by synthetic conditions and curing mechanism, are important technical materials and are irreplaceable in many areas, especially in thermal insulation, coating, adhesive, aeronautic utilities, and composite materials, because of their excellent thermal stability, high char yield, and good solvent resistance, etc [23]. The rapid growth of PF applications requires extensive research to improve their comprehensive properties. Moreover, it is demonstrated that many properties of PF can be easily improved by preparing PF composites [24]. Recently, researchers tried to prepare the PF nanocomposites by addition of nanoscale materials, including carbon naotubes [ 25 , 26], expanded graphite [23] and layered silicates $[27,28]$. There is a considerable amount of literature on the preparation and properties of polymermesoporous silica nanocomposites by using epoxy [10-13, 18, 22] and polyolefin resins [3, 6, 7] as polymer matrix. However, there are still few reports about the composite materials based on PF and mesoporous silica. In addition, though the researchers have paid much attention to the properties of the nanocomposites, systematic studies of structural variations of mesoporous silica in the process of the nanocomposites preparation have been rarely reported.

In this study, the novel SBA-15/PF composites were prepared via in situ condensation polymerization of phenol and formaldehyde in the presence of SBA-15. The mesoporous silica SBA-15 with large surface area, uniform hexagonal channels, large pore sizes and pore volume was synthesized by solgel method. In order to improve the interface compatibility between SBA-15 and PF matrix, surface modification of SBA-15 by coupling agent was conducted. Then, the modified SBA-15 was dispersed in phenol monomer by ultrasonic irradiation before polymerization. The structure of the SBA-15 by surface treatment and in situ polymerization methods and the effects of SBA-15 loading on the thermal properties of SBA-15/PF nanocomposites were investigated in detail.

\section{Experimental}

\subsection{Materials}

$\mathrm{EO}_{20} \mathrm{PO}_{70} \mathrm{EO}_{20}$ (where $\mathrm{EO}$ stands for ethylene oxide, $\mathrm{PO}$ for propylene oxide, Pluronic P123) was commercially obtained from Sigma-Aldrich Co., Ltd. (USA). Tetraethoxysilane (TEOS) used as silica source and $\gamma$-glycidyloxypropyltrimethoxysilane (GOTMS) used as coupling agent were purchased from Aladdin Chemistry Co. Ltd. (Shanghai, China). Phenol and formaldehyde monomers were ordered from Xilong Chemical Co. Ltd. (Guangdong, China). Oxalic acid was purchased from Shanghai Chemical Reagent Company (Shanghai, China) and used as catalytic agent. All chemicals were of reagentgrade and used as received without further purification.

\subsection{Synthesis of mesoporous silica materials}

Mesoporous silica materials, SBA-15, was firstly synthesized by templating with the $\mathrm{EO}_{20} \mathrm{PO}_{70} \mathrm{EO}_{20}$ triblock copolymers via a sol-gel process according to literature $[29,30]$. Typically, $2 \mathrm{~g}$ of Pluronic P123 and $37 \mathrm{wt} \% \mathrm{HCl}$ solution $(10 \mathrm{~mL})$ were dissolved in deionized water $(80 \mathrm{~g})$ with stirring at $35^{\circ} \mathrm{C}$ for $6 \mathrm{~h}$. Then $4.2 \mathrm{~g}$ of TEOS was dropped into the homogenous clear solution with stirring at $35^{\circ} \mathrm{C}$ for $24 \mathrm{~h}$. After that, the solution was then removed to an autoclave and hydrothermally treated by putting in a $100^{\circ} \mathrm{C}$ oven for another $24 \mathrm{~h}$. The obtained par- 
ticles were collected by filtration, washed 3times with deionized water, and dried at $60^{\circ} \mathrm{C}$ in air. The resulting products were calcined at $550 \mathrm{C}$ for $6 \mathrm{~h}$ to remove the template.

\subsection{Preparation of organically modified SBA-15 (SBA-15-GOTMS)}

The typical procedure for the preparation of organically modified SBA-15 is described as follows: the SBA-15 was dispersed in toluene under ultrasonic irradiation and stirring for $30 \mathrm{~min}$, and then the calculated amount of GOTMS was added to the mixture. The weight ratio between SBA-15 and GOTMS was $5: 1$. The mixture was refluxed at $120^{\circ} \mathrm{C}$ for $6 \mathrm{~h}$ under vigorous stirring. After the mixture was filtrated with Buchner funnel, the product was washed several times using acetone and dried in the vacuum oven at $60^{\circ} \mathrm{C}$.

\subsection{Preparation of SBA-15/PF nanocomposites} The SBA-15-GOTMS was first dispersed in phenol by ultrasonic irradiation and high temperature and high pressure treatment. In a typical procedure, the calculated amount of SBA-15-GOTMS was dispersed in phenol by ultrasonic irradiation in a beaker at $50^{\circ} \mathrm{C}$ for $1 \mathrm{~h}$. Subsequently, the mixture was transferred into an autoclave, sealing reactor, then put it into an oven at $200^{\circ} \mathrm{C}$ for $12 \mathrm{~h}$, following by natural cooling and reserve.

The novolac-type phenolic nanocomposites were synthesized in a $250 \mathrm{~mL}$ three-necked reactor equipped with a reflux condenser, stainless-steel stirrer, and thermometer. The reagents, phenol containing SBA-15, formaldehyde (in $37 \mathrm{wt} \%$ water solution) $(\mathrm{P}: \mathrm{F}$ molar ratio $=1.15: 1)$ and oxalic acid ( $2 \mathrm{wt} \%$ of phenol), were fed into a flask reactor. The mixture was first stirred under $85^{\circ} \mathrm{C}$ for $5 \mathrm{~h}$ in water bath, and then keep on reacting in order to remove the water and free phenol at $160-180^{\circ} \mathrm{C}$ for an additional $1 \mathrm{~h}$ under $0.03-0.05 \mathrm{MPa}$ pressure. When Weissenberg effect was observed, SBA-15/ PF nanocomposite was prepared [31]. The final products, i.e. SBA-15/PF nanocomposites which included various SBA-15 additives from 0 to $3 \mathrm{wt} \%$ were prepared as well.

\subsection{Preparation of E-SBA-15/PF hybrid materials}

SBA-15/PF nanocomposite containing $3 \mathrm{wt} \%$ SBA15 , was dissolved in a large volume of ethanol in a glass flask. The mixture was refluxed at $60^{\circ} \mathrm{C}$ for $12 \mathrm{~h}$ under stirring, and then centrifugal separated and washed for several times to remove most of the polymer from the external surface of the SBA-15 particles, and the residual sample named as E-SBA15/PF (extracted SBA-15/PF) was dried in vacuum at $80^{\circ} \mathrm{C}$ for $6 \mathrm{~h}$ before analyses [3]. Following the ESBA-15/PF was calcined at $500^{\circ} \mathrm{C}$ for $6 \mathrm{~h}$ to remove most of the organic compounds, the as-prepared powder materials were named after CE-SBA-15/PF. The experiment details of the process of hybrid materials are shown in Figure 1.

\subsection{Characterization}

X-ray powder diffraction (XRD) was carried out with a PANalytical X'Pert PRO X-ray Diffractometer (Holland). The X-ray beam was nickel-filtered $\mathrm{Cu}-\mathrm{K} \alpha$ radiation $(\lambda=0.154 \mathrm{~nm})$, the diffraction patterns being collected in the $2 \theta$ range $0.5-8.0^{\circ}$ at a scanning rate of $0.2 \% \mathrm{~min} . \mathrm{N}_{2}$ adsorption-desorption isotherms were obtained at $77 \mathrm{~K}$ using a Quantachrome NOVA 1200e gas-adsorption analyzer (USA). Before the adsorption measurements, all samples were outgassed for $12 \mathrm{~h}$ at $353 \mathrm{~K}$ in the degas port of the adsorption analyzer. The average pore radius and pore volume were determined by applying the BJH (Barrett, Joyner and Halenda) method. The specific surface area was calculated

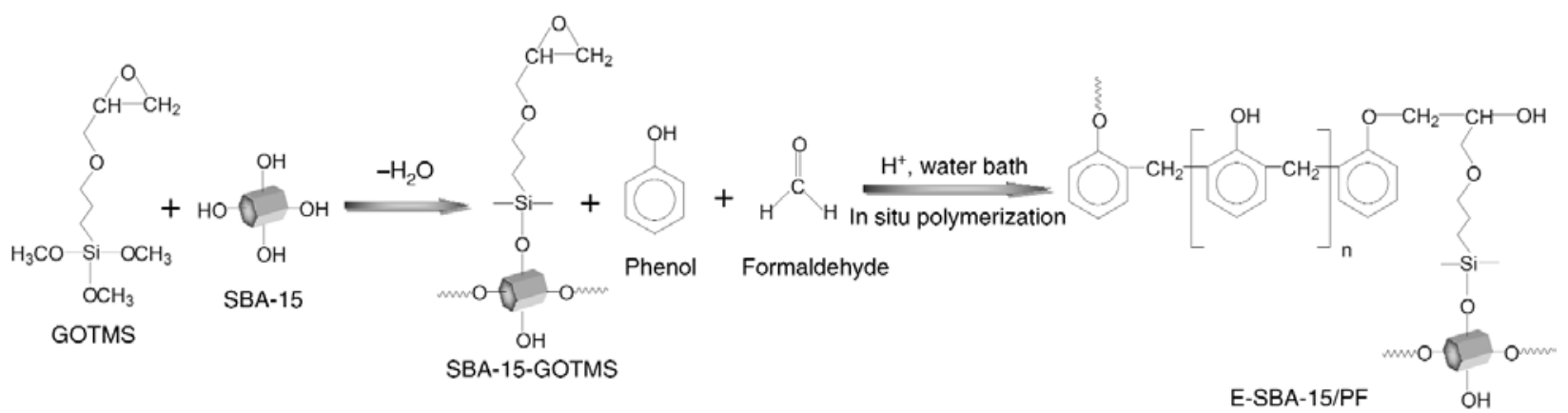

Figure 1. Scheme of possible reaction of the surface modification and in-situ polymerization 
using the BET (Brunauer, Emmet and Teller) model. Fourier-transform infrared spectroscopy (FTIR) spectra were recorded using a Nexus-470 FTIR spectrometer (Nicolet, USA), and the sample powder was pressed into a pellet with $\mathrm{KBr}$. Differential scanning calorimetry (DSC) was performed with DSC 204 (NETZSCH, Germany) between 10 and $100^{\circ} \mathrm{C}$ at a heating rate of $10^{\circ} \mathrm{C} / \mathrm{min}$ under a nitrogen atmosphere. Thermogravimetric analysis (TGA) was carried out on a STA 449C analyzer (NET$\mathrm{ZSCH}$, Germany) at a heating rate of $10^{\circ} \mathrm{C} / \mathrm{min}$ from 50 to $800^{\circ} \mathrm{C}$ under a nitrogen atmosphere. Scanning electron microscopy (SEM) observation was conducted with a JSM-6380 LV microscope (Japan) operating at $5 \mathrm{kV}$. Samples were sputter coated with a thin carbon layer under vacuum situation. Energy dispersive X-ray (EDX) spectroscopy image of the silicon element mapping was recorded using S-4800 scanning electron microscope (Hitachi, Japan). Transmission electron microscopy (TEM) was taken on JEM-2100 electron microscope (JEOL, Japan) operating at $120 \mathrm{kV}$. The sample powders were dispersed in ethanol under sonication, and then an aliquot was put on a copper grid and the solvent was evaporated in an oven.

\section{Results and discussion}

\subsection{Characterization of the surface modified SBA-15 and its nanocomposites}

In order to improve the interfacial adhesion between the SBA-15 and the PF matrix, the surface modification of SBA-15 was carried out. In this study, the GOTMS was used as coupling agent, which is capable of linking SBA-15 covalently to the PF resin by silylation of the mesoporous silica [12]. Then, SBA-

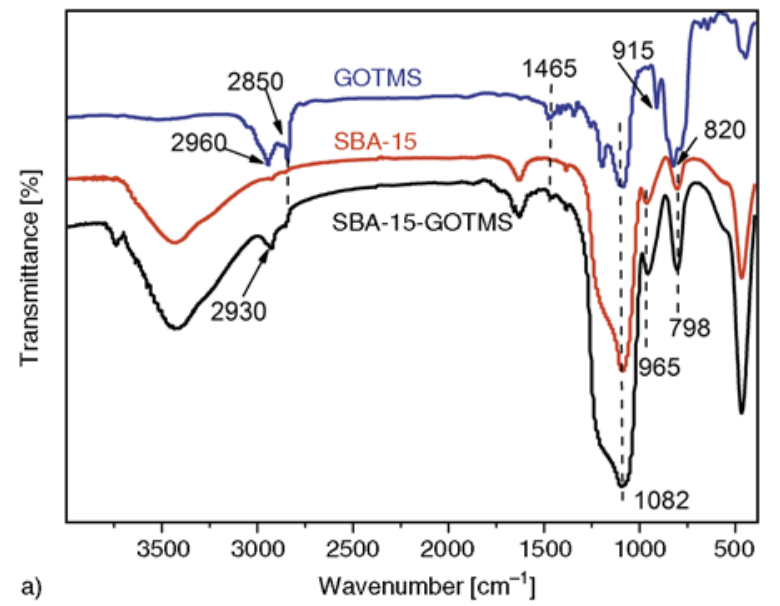

15/PF composites were prepared via in situ polymerization in the presence of SBA-15-GOTMS. The FTIR spectra of GOTMS, SBA-15 and SBA15-GOTMS are illustrated in Figure 2a. It can be seen that the GOTMS shows two epoxy group characteristic peaks at 915 and $820 \mathrm{~cm}^{-1}$, a weak $\mathrm{O}-\mathrm{CH}_{2}$ and $\mathrm{CH}_{3}$ bending vibrations at $1465 \mathrm{~cm}^{-1}$ [32] and two peaks at 2960 and $2850 \mathrm{~cm}^{-1}$ corresponding to the asymmetric and symmetric stretching vibrations of $\mathrm{C}-\mathrm{H}$ in $-\mathrm{CH}_{2}$ and $-\mathrm{CH}_{3}$, respectively. For unmodified SBA-15, a wide absorption peak at around $3450 \mathrm{~cm}^{-1}$ and a weak peak at $965 \mathrm{~cm}^{-1}$ may be assigned to $\mathrm{Si}-\mathrm{OH}$ stretching and bending vibrations, a strong absorption peak at $1082 \mathrm{~cm}^{-1}$ and a weak peak at $798 \mathrm{~cm}^{-1}$ associated with $\mathrm{Si}-\mathrm{O}-\mathrm{Si}$ asymmetric and symmetric stretching vibrations can be observed, respectively. However, the surface modified mesoporous silica materials (SBA-15GOTMS) possess many characteristic absorption peaks derived from SBA-15 and GOTMS. It can be clearly seen that both $\mathrm{Si}-\mathrm{O}-\mathrm{Si}$ and $\mathrm{Si}-\mathrm{OH}$ characteristic absorption peaks are kept, and the epoxy group peak at 915 and $820 \mathrm{~cm}^{-1}$ are overlapped by a series of intensive peaks at $798-1082 \mathrm{~cm}^{-1}$ which belong to $\mathrm{Si}-\mathrm{O}-\mathrm{Si}$ and $\mathrm{Si}-\mathrm{OH}$ [12]. Additionally, the adsorptions peak shifts from 2960 to $2930 \mathrm{~cm}^{-1}$, which can be attributed to asymmetric stretching vibrations of $\mathrm{C}-\mathrm{H}$, and it implies the $-\mathrm{CH}_{3}$ is absent in the SBA-15-GOTMS. All these results suggest that the GOTMS has been attached to the surface of SBA-15 through condensation between the silanol groups of hydrolyzed GOTMS and SBA-15. Figure $2 \mathrm{~b}$ shows the FTIR spectra of PF, SBA-15GOTMS and E-SBA-15/PF hybrid materials. The E-SBA-15/PF exhibit many characteristic absorp-



Figure 2. FTIR spectra of GOTM, SBA-15, and SBA-15-GOTMS (a), PF, SBA-15-GOTMS and E- SBA-15/PF (b) 
tion peaks both of PF and SBA-15-GOTMS. Among them, the peaks at $1400-1600 \mathrm{~cm}^{-1}$, corresponding to the phenyl rings, and $3030 \mathrm{~cm}^{-1}$, corresponding to $\mathrm{C}-\mathrm{H}$ stretching vibration of phenyl rings, are presented in the E-SBA-15/PF hybrid materials as well as pure PF. A strong absorption peak at $1082 \mathrm{~cm}^{-1}$ is attributed to the $\mathrm{Si}-\mathrm{O}-\mathrm{Si}$ stretch vibration appears in the FTIR spectra of the SBA-15-GOTMS and E-SBA-15/PF hybrid materials. But the peak at $1230 \mathrm{~cm}^{-1}$, corresponding to stretching vibration of phenolic $\mathrm{C}-\mathrm{O}-\mathrm{C}$ [33], is overlapped by an intensive peak at $1000-1300 \mathrm{~cm}^{-1}$ belonging to the $\mathrm{Si}-\mathrm{O}-\mathrm{Si}$ characteristic peak. The results demonstrate that SBA-15-GOTMS has been covalently bonded with $\mathrm{PF}$ chain during in situ polymerization. The presence of covalent conjugation could improve the interfacial adhesion between SBA-15-GOTMS and PF.

The nitrogen adsorption-desorption isotherms along with the corresponding pore-size distribution curves of SBA-15, SBA-15-GOTMS and E-SBA-15/PF are shown in Figure 3. The SBA-15 exhibits a classical Langmuir IV-type isotherm with $\mathrm{H}_{1}$ type vertical hysteresis loop, and the capillary condensation occurs at a high relative pressure, which is conformable to the literature [29]. In addition, the $\mathrm{H}_{1}$ type hysteresis loop of the SBA-15 is relatively wide. We think this may be caused by a wide distribution of the pore volume SBA-15. The curves of SBA-15-GOTMS and E-SBA-15/PR show rarely adsorption steps and $\mathrm{H}_{1}$ type hysteresis loop (Figure $3 \mathrm{a}$ ), and the pore volumes decreased from 1 to 0.71 and $0.04 \mathrm{~cm}^{3} \cdot \mathrm{g}^{-1}$, respectively. The detailed structural parameters of the SBA-15 and its hybrid materials are listed in Table 1. The results indicate that GOTMS has been conjugated to SBA-15 by

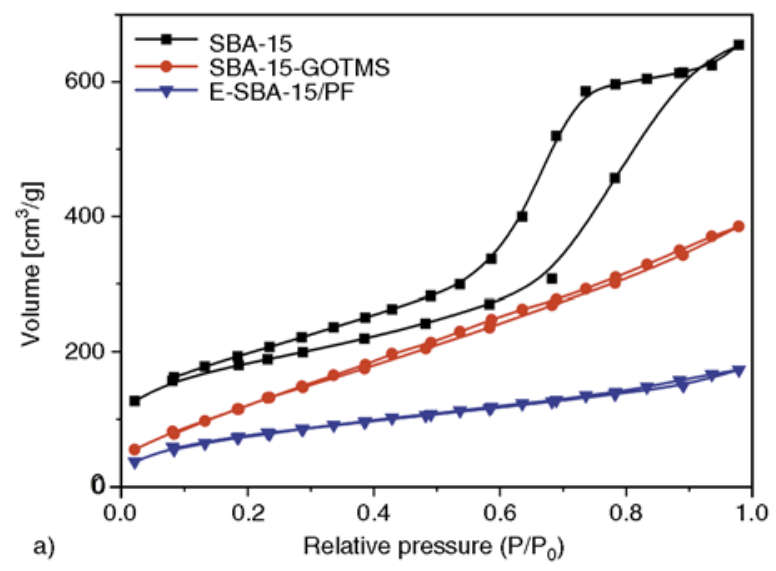

Table 1. Textural parameters of SBA-15, SBA-15-GOTMS and E-SBA-15 /PF

\begin{tabular}{|l|c|c|c|}
\hline \multicolumn{1}{|c|}{ Material } & $\begin{array}{c}\text { BET } \\
\text { Surface area } \\
{\left[\mathbf{m}^{2} / \mathbf{g}\right]}\end{array}$ & $\begin{array}{c}\text { Total pore } \\
\text { volume } \\
{\left[\mathbf{c m}^{\mathbf{3}} / \mathbf{g}\right]}\end{array}$ & $\begin{array}{c}\text { Average pore } \\
\text { diameter } \\
{[\mathbf{n m}]}\end{array}$ \\
\hline SBA-15 & 707 & 1.00 & 6.6 \\
\hline SBA-15-GOTMS & 619 & 0.71 & 2.0 \\
\hline E-SBA-15/PF & 46 & 0.04 & 1.6 \\
\hline
\end{tabular}

chemical bond, and the condensation polymerization of PF has been successfully carried out inside the channels of SBA-15, leading to occupation most of channel space of SBA-15. Therefore, the pore volume of E-SBA-15/PF is very small after the polymerization. Moreover, the three samples exhibited great differences of the distribution of pore size. The distribution of pore size of SBA-15-GOTMS and E-SBA-15/PF are broader than that of SBA-15 (Figure 3b), which may be attributed to the fact that the mesoporous channels were plugged up in various extents after the coupled treatment and polymerization occurred [4, 34]. On the other hand, this also indicated the successful reaction within the channels of SBA- 15 .

Figure 4 shows the XRD patterns of mesostructure silica. There are three well-defined peaks at $2 \theta$ values between 0.5 and $8^{\circ}$ which can be indexed as (100), (110), and (200) Bragg reflections, suggested the typical hexagonal (p6mm) SBA-15 [30]. After treating SBA-15 by GOTMS, the XRD pattern of the resulted SBA-15-GOTMS shows the p6mm structural feature as well, although the diffraction peaks increased slightly in $2 \theta$ values. The three characteristic XRD peaks of SBA-15-GOTMS can still be observed after treatment by coupling agent, demonstrate that hexagonal SBA-15 was undam-

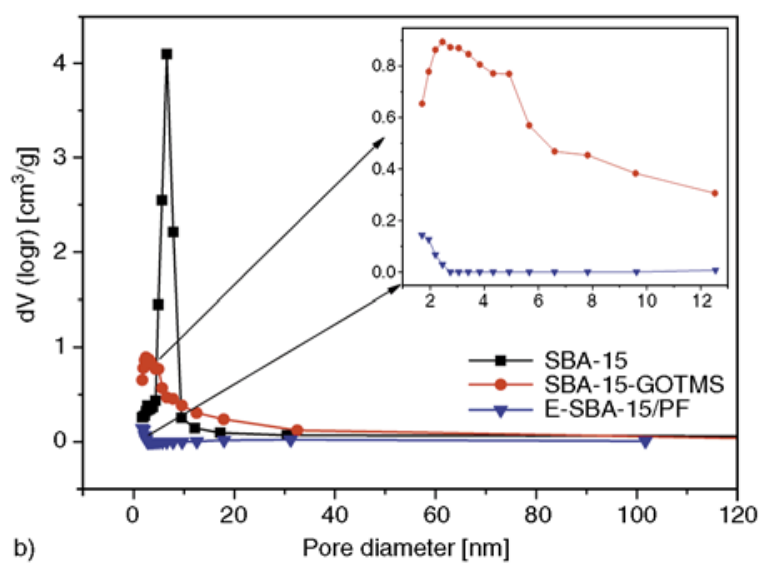

Figure 3. Nitrogen sorption isotherms (a) and pore diameter distribution (b) of SBA-15, SBA-15-GOTMS and E-SBA$15 / \mathrm{PF}$ 


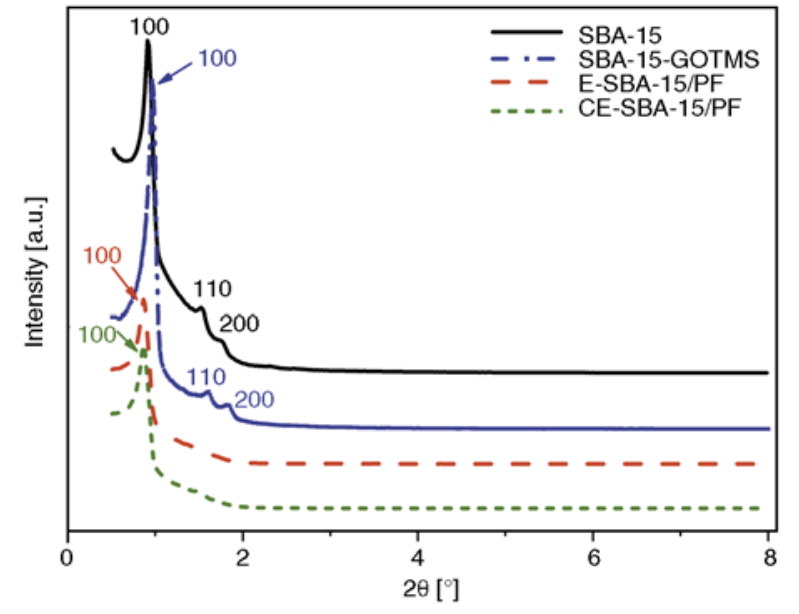

Figure 4. X-ray powder diffraction of SBA-15, SBA-15GOTMS, E-SBA-15/PF and CE-SBA-15/PF

aged. From the XRD pattern of E-SBA-15/PF and CE-SBA-15/PF, we can see that the intensity of (100) diffraction peak was distinctly decreased, meanwhile the peaks at higher angles are not observed, suggesting that the ordered structure of SBA-15 has been little changed in short-range but great changed in long-range. This may be explained by the following reasons: the SBA-15 were uniformly dispersed throughout the polymer matrix during the in situ polymerization process, and meanwhile interpenetrating organic-inorganic network structure may be formed, leading to the decrease of long range regularity of SBA-15.

Thermogravimetric analysis (TGA) curves of SBA15, SBA-15-GOTMS, PF and E-SBA-15/PF are shown in Figure 5. It can be seen that only $1.8 \mathrm{wt} \%$ weight loss was found for unmodified SBA-15 below $800^{\circ} \mathrm{C}$, while 13.48 and $34.02 \mathrm{wt} \%$ weight loss were observed between $50-800^{\circ} \mathrm{C}$ for SBA-15GOTMS and E-SBA-15/PF, respectively. All these

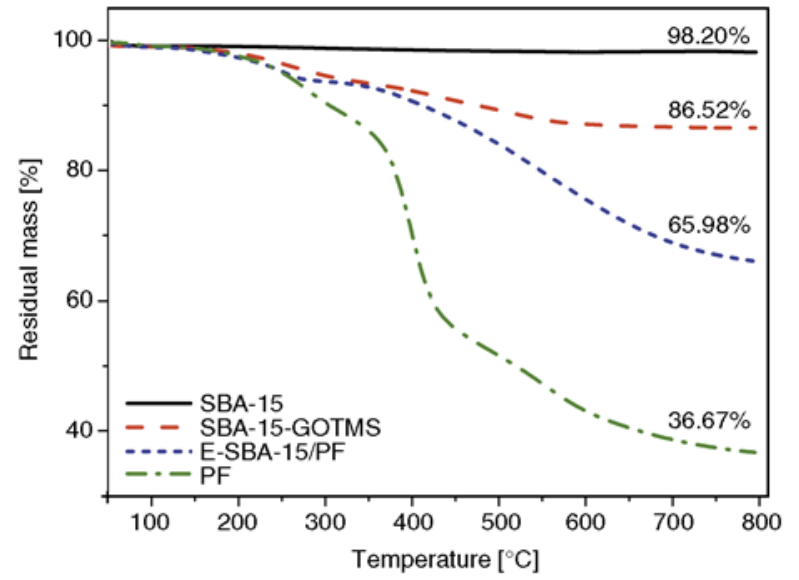

Figure 5. TGA thermograms of SBA-15, SBA-15-GOTMS, $\mathrm{PF}$ and E-SBA-15/PF results demonstrate that the GOTMS has been successfully grafted onto the surface of the SBA-15 and the chemical reaction has occurred between SBA-15-GOTMS and PF resin via in situ polymerization. From the TGA curves, the reacting masses of GOTMS grafted onto SBA-15 can be approximately calculated, which is $11.68 \mathrm{wt} \%$. Taking into consideration that the carbon residue of $\mathrm{PF}$ is up to $36.67 \mathrm{wt} \%$ at $800^{\circ} \mathrm{C}$, the masses of $\mathrm{PF}$ reacted with SBA-15-GOTMS (RMp) during in situ polymerization can be estimated using the Equation (1) [35]:

$R M_{\mathrm{p}}=\frac{W_{\mathrm{SBA}-15-\mathrm{GOTMS}, \mathrm{T}}-W_{\mathrm{E}-\mathrm{SBA}-15 / \mathrm{PF}, \mathrm{T}}}{1-W_{\mathrm{PF}, \mathrm{T}}}$

where $R M_{\mathrm{p}}$ is the masses of PF reacted with SBA15-GOTMS by in situ polymerization; $W_{\mathrm{PF}, \mathrm{T}}$, $W_{\text {SBA-15-GOTMS,T }}$ and $W_{\mathrm{E}-\mathrm{SBA}-15 / \mathrm{PF}, \mathrm{T}}$ are the residual weight percent of PF, SBA-15-GOTMS and ESBA-15/PF, respectively, at temperature $T$. The value of $R M_{\mathrm{p}}$ was $32.43 \mathrm{wt} \%$ of SBA-15 according to the Equation (1). Meanwhile, it could be seen from the TGA curves of E-SBA-15/PF that a platform arose at $250-350^{\circ} \mathrm{C}$ as compared with $\mathrm{PF}$, suggested that the thermal stability of E-SBA$15 / \mathrm{PF}$ was greatly enhanced, which confirmed that there was a strong interaction between SBA-15GOTMS and PF resin.

\subsection{Morphology}

Figure 6 shows the SEM micrographs of the SBA15, SBA-15-GOTMS and E-SBA-15/PF. It can be clearly observed that the SBA-15 consist of many rope-like shapes with relatively uniform sizes of 1$2 \mu \mathrm{m}$, which are aggregated each other and form clusters. The morphology characteristic of SBA-15 is well in accordance with previous literature [29]. After surface modification, SBA-15-GOTMS shows the similar particle morphology as SBA-15, suggested the high stability of ordered structure of SBA-15. Figure 6c shows the SEM image of ESBA-15/PF (the extracted SBA-15/PF), which reveals that both the surface and interface of SBA15 attached some PF resins but the SBA-15 could still be distinguished easily. Furthermore, the larger orderly agglomerates of the SBA- 15 have been broken, and some holes among SBA-15 appeared resulting from the extracted PF matrix. The lose of orderly structure of the SBA-15 seen from the SEM image and the above XRD analysis may be attributed to 


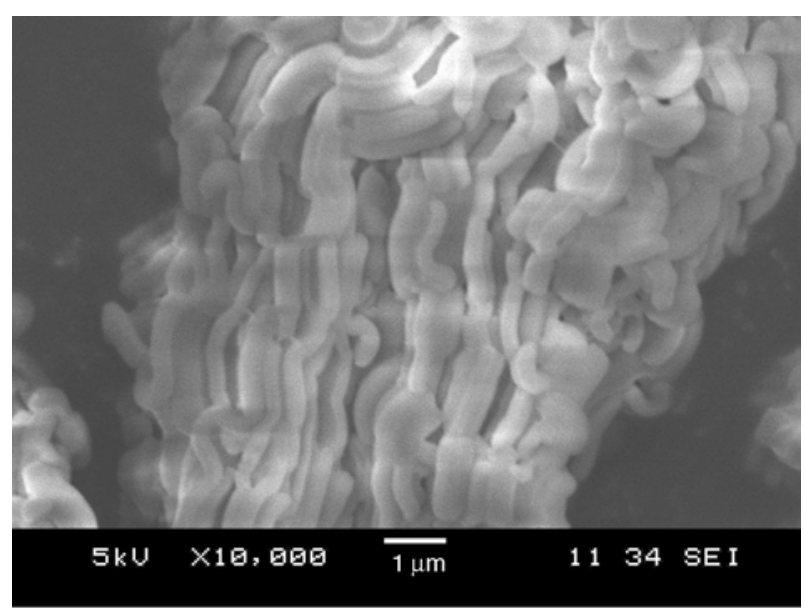

a)

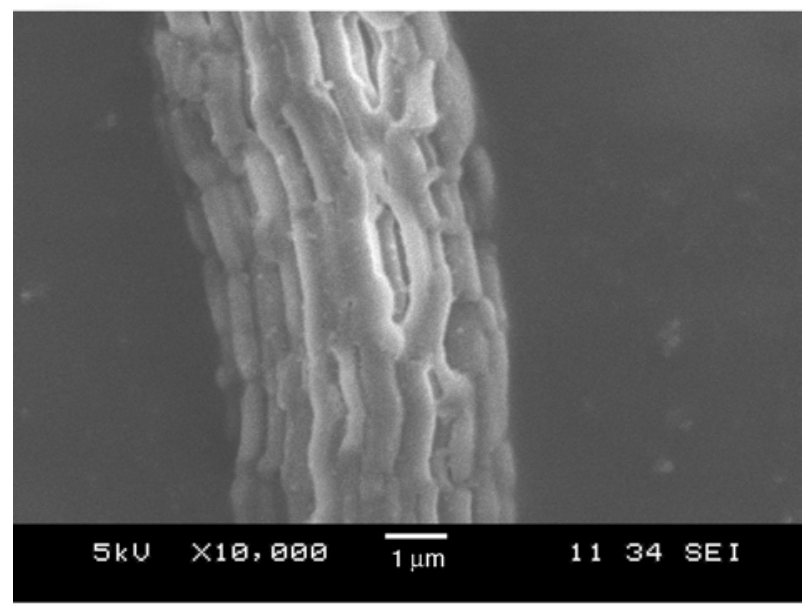

b)

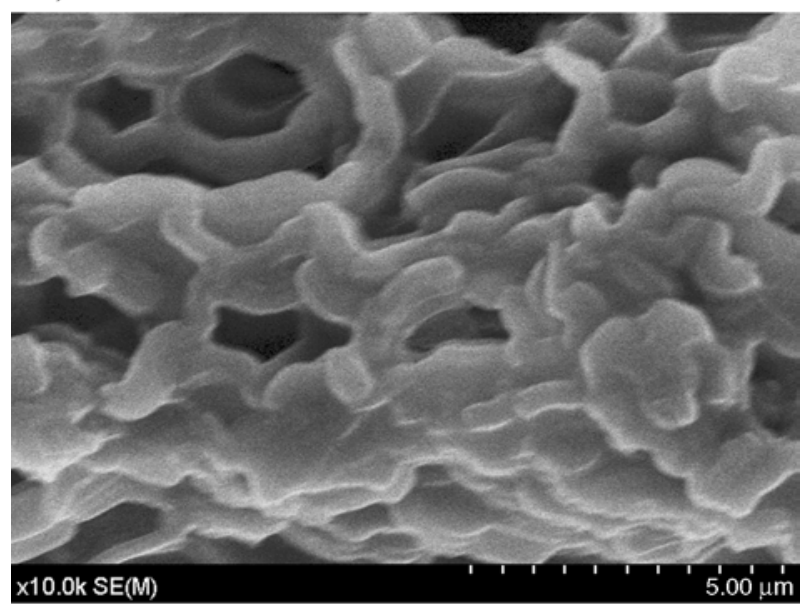

c)

Figure 6. SEM images of SBA-15 (a), SBA-15-GOTMS (b) and E-SBA-15/PF (c)

the formation of chemical bonds Between SBA-15 and PF during in situ polymerization. This effect could contribute to the homogeneous dispersion of mesoporous silica in the PF matrix.

To further observe the variation of morphology of SBA-15 before and after in situ polymerization, the TEM images of the SBA-15 and CE-SBA-15/PF
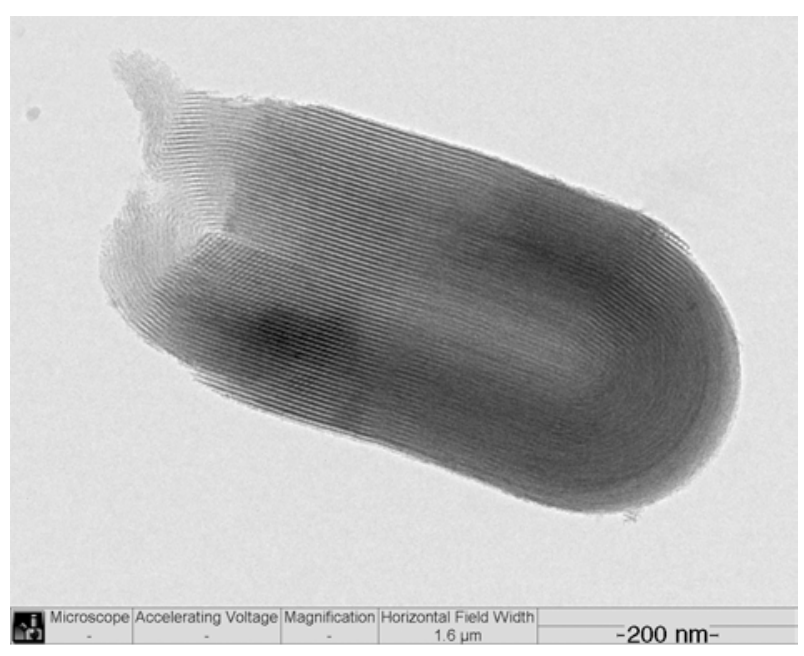

a)

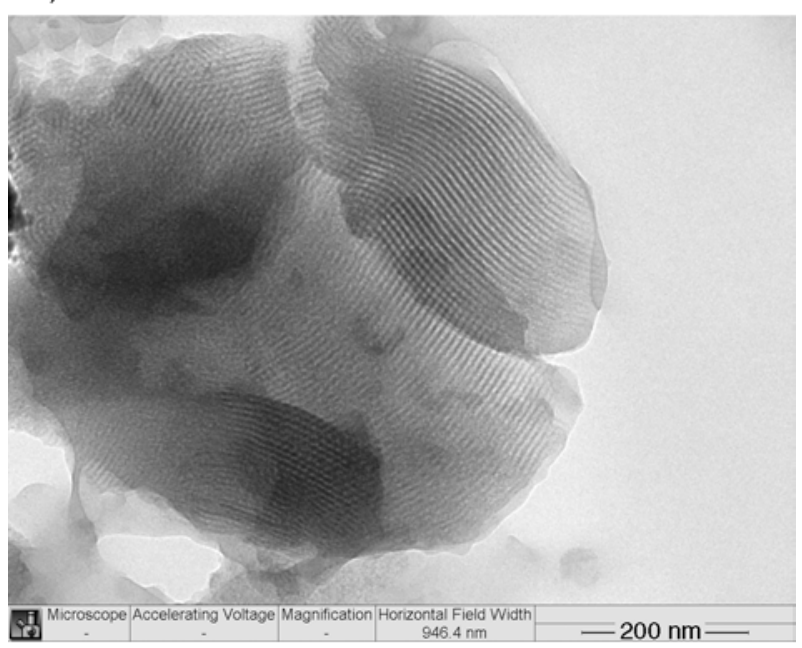

b)

Figure 7. TEM images of the SBA-15 (a) and CE-SBA$15 / \mathrm{PF}$ (b) hybrid material

are presented as shown in Figure 7. The images of the SBA-15 show large domains of well-ordered structure with 1D channels, which are similar to results reported in literatures [29-30]. It can be seen from Figure $7 \mathrm{~b}$ that the large domain regularity of CE-SBA-15/PF has decreased, and there are still the 1D channels of mesoporous materials in the relatively small domain, which is consistent with the XRD analysis. This results can further confirm that the effect of the in situ polymerization on the ordered structure of SBA-15.

In order to further study the dispersion of SBA-15 in the PF matrix, EDX mapping of SBA-15/PF composite materials were carried out. Figure 8 show $\mathrm{Si}$ mapping of SBA-15/PF composite materials with various contents of SBA-15. It can be clearly seen that lots of white spots associated with silicon atoms were uniformly distributed in black background 


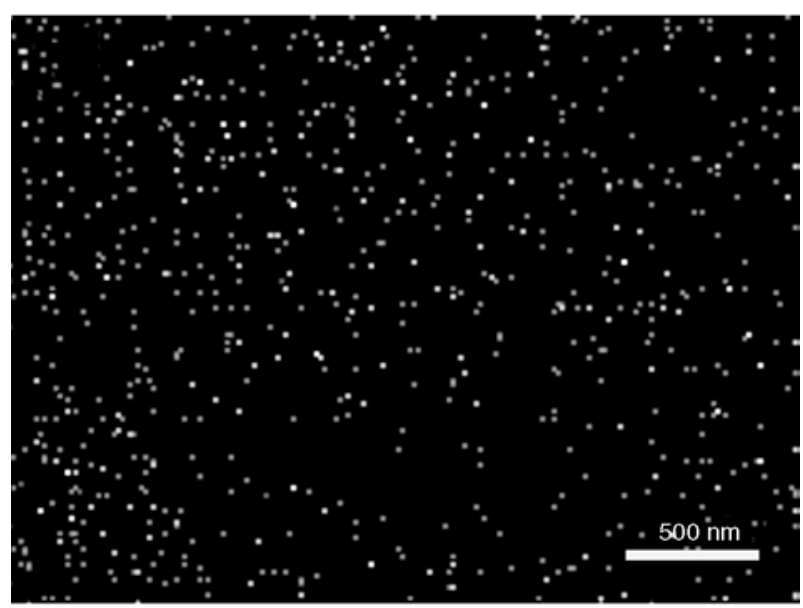

a)

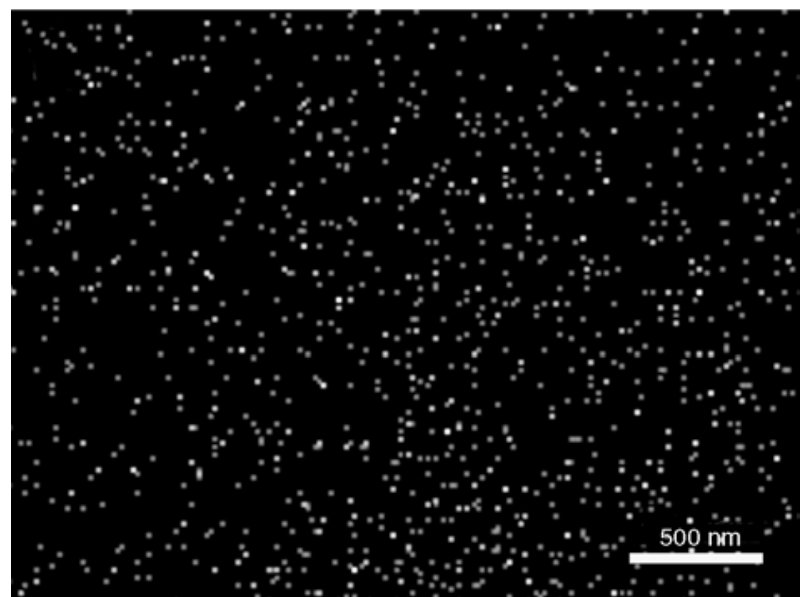

b)

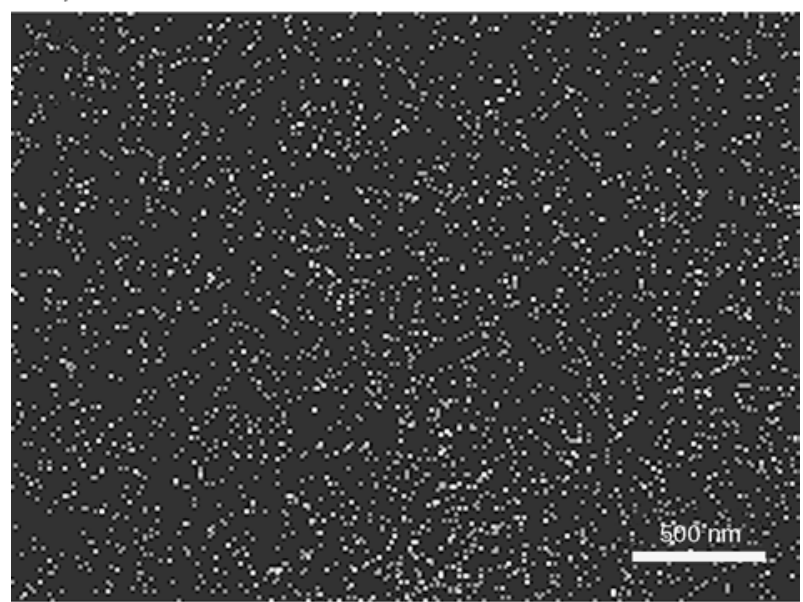

c)

Figure 8. Si-mapping images studies of EDX on SBA$15 / \mathrm{PF}$ composites (a) $1 \%$, (b) $2 \%$ and (c) $3 \%$

associated with polymer matrix at $\times 20000$ magnification (scale bar $=500 \mathrm{~nm}$ ), indicating that the SBA15 was well dispersed throughout the PF matrix. Moreover, with the increasing of SBA-15 content, the density of silicon atoms was distinctly increased.

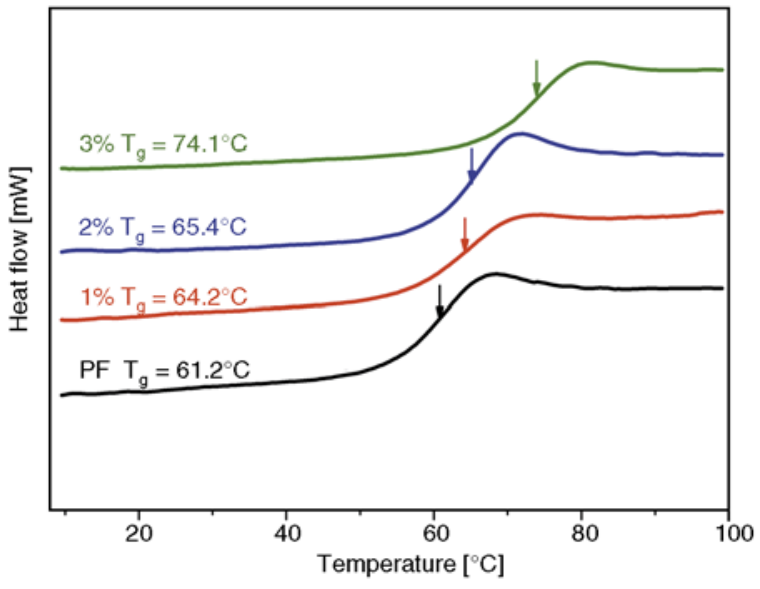

Figure 9. DSC curves and $T_{\mathrm{g}}$ values for PF and SBA-15/PF nanocomposites

\subsection{Thermal properties}

The thermal properties of polymer nanocomposites have drawn much attention because of the demand of many application fields of the nanocomposites. Furthermore, the improvement of the thermal properties of polymer nanocomposites can also demonstrate the uniform dispersion of fillers and the presence of intensive interactions between fillers and polymers to a certain extent. Figure 9 shows the variation of the glass transition temperature $\left(T_{\mathrm{g}}\right)$ with SBA-15 loading as determined by conventional DSC for the unmodified and modified PF. $T_{\mathrm{g}}$ of the pure $\mathrm{PF}$ is about $61.2^{\circ} \mathrm{C}$, whereas all the SBA15/PF nanocomposite show substantially higher $T_{\mathrm{g}}$ values than that of pure PF. Moreover, $T_{\mathrm{g}}$ of nanocomposites is enhanced with the increase of SBA15 contents. It is worth noting that the $T_{\mathrm{g}}$ of the nanocomposite containing $3 \mathrm{wt} \%$ SBA- 15 is increased by $12.9^{\circ} \mathrm{C}$ in contrast to pure PF. The significant enhancement of $T_{\mathrm{g}}$ may be attributed to the strong interactions between the polymer chains and SBA15 which was formed by in situ polymerization. More importantly, the in situ polymerization of PF monomers within the pore channels of SBA-15, may trap some polymer chains or fractions. These interactions between PF and SBA-15 will impede the movement of polymer chains and result in the increases of $T_{\mathrm{g}}$ significantly with addition of SBA-15. Figure 10 and Table 2 show the results of the thermal stabilities of the SBA-15, pure PF and its nanocomposites by using TGA in the range of $50-800^{\circ} \mathrm{C}$. 
Table 2. Thermal stabilities of SBA-15, PF and SBA-15/PF nanocomposites

\begin{tabular}{|l|c|c|c|}
\hline \multicolumn{1}{|c|}{ Material } & $\begin{array}{c}\mathbf{T}_{\mathbf{d}, \mathbf{5}} \\
{\left[{ }^{\circ} \mathbf{C}[\right.}\end{array}$ & $\begin{array}{c}\mathbf{T}_{\mathbf{d}, \mathbf{1 0}} \\
{\left[{ }^{\circ} \mathbf{C}[\right.}\end{array}$ & $\begin{array}{c}\text { Char yield at } \mathbf{~ 8 0 0}^{\circ} \mathbf{C} \\
{[\mathbf{w t} \mathbf{0}]}\end{array}$ \\
\hline pure PF & 263 & 312 & 37.7 \\
\hline SBA-15 & - & - & 98.2 \\
\hline $1 \mathrm{wt} \% \mathrm{SBA}-15 / \mathrm{PF}$ & 266 & 317 & 39.1 \\
\hline $2 \mathrm{wt} \% \mathrm{SBA}-15 / \mathrm{PF}$ & 279 & 337 & 42.0 \\
\hline $3 \mathrm{wt} \% \mathrm{SBA}-15 / \mathrm{PF}$ & 364 & 380 & 49.7 \\
\hline
\end{tabular}

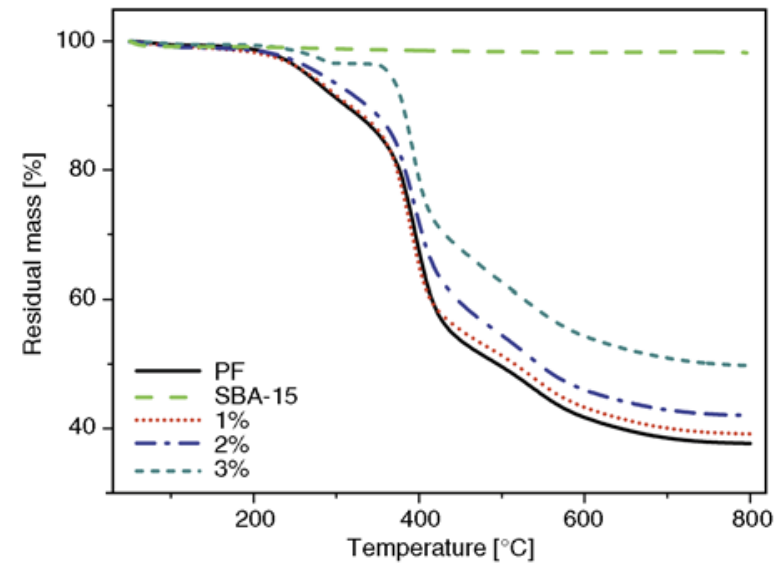

Figure 10. TGA curves of SBA-15, PF and SBA-15/PF nanocomposites

Thermal stabilities of the nanocomposites are expressed by their 5 and $10 \%$ weight loss decomposition temperatures, $T_{\mathrm{d}, 5}$ and $T_{\mathrm{d}, 10}$. As expected, the SBA-15/PF nanocomposites show better thermal stability than the pure PF. Moreover, the $T_{\mathrm{d}, 5}$ and $T_{\mathrm{d}, 10}$ of the nanocomposites increase with respect to the amount of SBA-15, for instance, the $T_{\mathrm{d}, 5}$ and $T_{\mathrm{d}, 10}$ of SBA-15/PF nanocomposites with 1 and $3 \mathrm{wt} \%$ SBA- 15 increases from 263 and $312^{\circ} \mathrm{C}$ (neat $\mathrm{PF})$ to 266 and $317^{\circ} \mathrm{C}(1 \mathrm{wt} \%)$ and 364 and $380^{\circ} \mathrm{C}$ (3 $\mathrm{wt}^{\%} \%$ ), respectively. Meanwhile, for the TGA curves of SBA-15/PF nanocomposites with $3 \mathrm{wt} \%$ SBA-15 loading, a platform appeared in the range of $300-350^{\circ} \mathrm{C}$. In addition, the char yield of the nanocomposites increased with the increase of SBA15 content, and is higher than its SBA-15 content (Table 2), because the introduction of SBA-15 hindered the production of gaseous product and resulted in increase of the char retention. The obvious enhancement of the thermal stability of the nanocomposites can be attributed to the presence of strong interaction between SBA-15 and polymer matrix. The modified SBA-15 nanoparticles have abundant GOTMS on their surface and internal pore channel surface, and thus chemical bonding between the PF chains and SBA-15 can be formed after in situ polymerization. Hence, it's believed that PF chains not only bonded to the surface of the SBA-15, but also penetrated through the pore channel and were tethered to the channel surface. The favorable interaction leads to homogeneous disperse of SBA-15 in the PF matrix.

\section{Conclusions}

The PF nanocomposites containing SBA-15 type mesoporous silica modified by GOTMS have successfully been prepared via in situ polymerization. From the FTIR and TGA analysis, the GOTMS was chemically grafted onto the surface of SBA-15 and there were chemical reaction between SBA-15GOTMS and PF chains during in situ polymerization. In addition, the variation of the textural parameters of SBA-15 was studied during the reaction process by XRD, $\mathrm{N}_{2}$ adsorption-desorption, SEM, and TEM. The results show that both surface modification and in situ polymerization treatments have great influence on the textural parameters of SBA15.

We also investigated the effect of SBA-15 loading on glass transition temperatures and thermal stability of the SBA-15/PF nanocomposites. With the increase of the SBA-15 content, the $T_{\mathrm{d}, 5}, T_{\mathrm{d}, 10}$ and $T_{\mathrm{g}}$ of PF nanocomposites were gradually improved as compared to that of the neat PF. It is especially exciting that the $T_{\mathrm{g}}$ and $T_{\mathrm{d}, 10}$ of the PF nanocomposites were increased by 12.9 and $68^{\circ} \mathrm{C}$ at $3 \mathrm{wt} \%$ SBA-15 loading, respectively. From the analysis of EDX mapping of SBA-15/PF composite materials, the uniform dispersion of the SBA-15 in the PF matrix may be the cause for the enhancement of thermal properties. Because of the excellent thermal properties of the new nanocomposites, it has great potential for friction composite materials and electronic packaging materials applications.

\section{Acknowledgements}

The authors would like to acknowledge National Natural Science Foundation of China (51163003), Guangxi Natural Science Foundation of China (2010GXNSFA013030 and 2012GXNSFBA053155) for financial support. 


\section{References}

[1] Joseph J., Tseng C-Y., Pan C-J., Chen H-M., Lin C-W., Pillai K. C., Hwang B-J.: Growing well-defined monodispersed silica in polyimide host membranes using a surfactant assisted sol-gel process. Polymer, 51, 56635668 (2010).

DOI: 10.1016/j.polymer.2010.09.064

[2] Zou H., Wu S., Shen J.: Polymer/silica nanocomposites: Preparation, characterization, properties, and applications. Chemical Reviews, 108, 3893-3957 (2008). DOI: $10.1021 / \mathrm{cr} 068035 \mathrm{q}$

[3] Run M. T., Wu S. Z., Zhang D. Y., Wu G.: A polymer/mesoporous molecular sieve composite: Preparation, structure and properties. Materials Chemistry and Physics, 105, 341-347 (2007).

DOI: $10.1016 /$ j.matchemphys.2007.04.070

[4] Choi M., Kleitz F., Liu D., Lee H. Y., Ahn W-S., Ryoo R.: Controlled polymerization in mesoporous silica toward the design of organic-inorganic composite nanoporous Materials. Journal of the American Chemical Society, 127, 1924-1932 (2005).

DOI: $10.1021 / \mathrm{ja} 044907 \mathrm{z}$

[5] Ariga K., Vinu A., Yamauchi Y., Ji Q., Hill J. P.: Nanoarchitectonics for mesoporous materials. Bulletin of the Chemical Society of Japan, 85, 1-32 (2012). DOI: $10.1246 /$ bcsj.20110162

[6] Zhang F-A., Lee D-K., Pinnavaia T. J.: PMMA/mesoporous silica nanocomposites: Effect of framework structure and pore size on thermomechanical properties. Polymer Chemistry, 1, 107-113 (2010).

DOI: 10.1039/B9PY00232d

[7] Zhang F-A., Lee D-K., Pinnavaia T. J.: PMMA-mesocellular foam silica nanocomposites prepared through batch emulsion polymerization and compression molding. Polymer, 50, 4768-4774 (2009).

DOI: $10.1016 /$ j.polymer.2009.08.007

[8] Shi J-L., Hua Z-L., Zhang L-X.: Nanocomposites from ordered mesoporous materials. Journal of Materials Chemistry, 14, 795-806 (2004).

DOI: $10.1039 / \mathrm{B} 315861 \mathrm{f}$

[9] Fujiwara M., Shiokawa K., Zhu Y.: Preparation of mesoporous silica/polymer sulfonate composite materials. Journal of Molecular Catalysis A: Chemical, 264, 153-161 (2007).

DOI: $10.1016 /$ j.molcata.2006.09.016

[10] Suzuki N., Kiba S., Yamauchi Y.: Low dielectric property of novel mesoporous silica/polymer composites using smart molecular caps: Theoretical calculation of air space encapsulated inside mesopores. Microporous and Mesoporous Materials, 138, 123-131 (2011). DOI: $10.1016 /$ j.micromeso.2010.09.020

[11] Lu S., Chun W., Yu J., Yang X.: Preparation and characterization of the mesoporous $\mathrm{SiO}_{2}-\mathrm{TiO}_{2} /$ epoxy resin hybrid materials. Journal of Applied Polymer Science, 109, 2095-2102 (2008).

DOI: $10.1002 / a p p .27856$
[12] Lin J. J., Wang X. D.: New type of low-dielectric composite based on $o$-cresol novolac epoxy resin and mesoporous silicas: Fabrication and performances. Journal of Materials Science, 43, 4455-4465 (2008).

DOI: $10.1007 / \mathrm{s} 10853-008-2655-5$

[13] Lin J., Wang X.: Preparation, microstructure, and properties of novel low- $\kappa$ brominated epoxy/mesoporous silica composites. European Polymer Journal, 44, 1414-1427 (2008).

DOI: $10.1016 /$ j.eurpolymj.2008.02.022

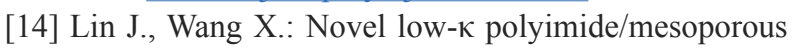
silica composite films: Preparation, microstructure, and properties. Polymer, 48, 318-329 (2007).

DOI: $10.1016 /$ j.polymer.2006.10.037

[15] Yu X., Ding S., Meng Z., Liu J., Qu X., Lu Y., Yang Z.: Aerosol assisted synthesis of silica/phenolic resin composite mesoporous hollow spheres. Colloid and Polymer Science, 286, 1361-1368 (2008).

DOI: $10.1007 / \mathrm{s} 00396-008-1904-0$

[16] Lee T., Park S. S., Jung Y., Han S., Han D., Kim I., Ha C-S.: Preparation and characterization of polyimide/ mesoporous silica hybrid nanocomposites based on water-soluble poly(amic acid) ammonium salt. European Polymer Journal, 45, 19-29 (2009). DOI: 10.1016/j.eurpolymj.2008.09.022

[17] Hu J., Gu A., Liang G., Zhuo L., Yuan L.: Preparation and properties of mesoporous silica/bismaleimide/ diallylbisphenol composites with improved thermal stability, mechanical and dielectric properties. Express Polymer Letters, 5, 555-568 (2011).

DOI: 10.3144 /expresspolymlett.2011.54

[18] Suzuki N., Kiba S., Yamauchi Y.: Fabrication of mesoporous silica KIT-6/polymer composite and its low thermal expansion property. Materials Letters, 65, 544547 (2011). DOI: $10.1016 /$ j.matlet.2010.10.027

[19] Suzuki N., Kiba S., Yamauchi Y.: Bimodal filler system consisting of mesoporous silica particles and silica nanoparticles toward efficient suppression of thermal expansion in silica/epoxy composites. Journal of Materials Chemistry, 21, 14941-14947 (2011).

DOI: 10.1039/C1JM12405F

[20] Suzuki N., Kiba S., Yamauchi Y.: Fabrication of mesoporous silica/polymer composites through solvent evaporation process and investigation of their excellent low thermal expansion property. Physical Chemistry Chemical Physics, 13, 4957-4962 (2011). DOI: $10.1039 / \mathrm{C0CP} 02071 \mathrm{~K}$

[21] Suzuki N., Kiba S., Kamachi Y., Miyamoto N., Yamauchi Y.: Mesoporous silica as smart inorganic filler: preparation of robust silicone rubber with low thermal expansion property. Journal of Materials Chemistry, 21, 5338-5344 (2011). DOI: $10.1039 / \mathrm{C} 0 J M 03767 \mathrm{~B}$ 
[22] Romanes M. C., D’Souza N. A., Coutinho D., Balkus K. J., Scharf T. W.: Surface and subsurface characterization of epoxy-mesoporous silica composites to clarify tribological properties. Wear, 265, 88-96 (2008). DOI: 10.1016/j.wear.2007.08.022

[23] Zhang X., Shen L., Xia X., Wang H., Du Q.: Study on the interface of phenolic resin/expanded graphite composites prepared via in situ polymerization. Materials Chemistry and Physics, 111, 368-374 (2008).

DOI: $10.1016 /$ j.matchemphys.2008.04.028

[24] Abdalla M. O., Ludwick A., Mitchell T.: Boron-modified phenolic resins for high performance applications. Polymer, 44, 7353-7359 (2003).

DOI: $10.1016 /$ j.polymer.2003.09.019

[25] Liu L., Ye Z.: Effects of modified multi-walled carbon nanotubes on the curing behavior and thermal stability of boron phenolic resin. Polymer Degradation and Stability, 94, 1972-1978 (2009).

DOI: 10.1016/j.polymdegradstab.2009.07.022

[26] Ye C., Gong Q-M., Lu F-P., Liang J.: Preparation of carbon nanotubes/phenolic-resin-derived activated carbon spheres for the removal of middle molecular weight toxins. Separation and Purification Technology, 61, 9-14 (2008).

DOI: 10.1016/j.seppur.2007.09.021

[27] Kaynak C., Tasan C. C.: Effects of production parameters on the structure of resol type phenolic resin/layered silicate nanocomposites. European Polymer Journal, 42, 1908-1921 (2006).

DOI: 10.1016/j.eurpolymj.2006.03.008

[28] Tasan C. C., Kaynak C.: Mechanical performance of resol type phenolic resin/layered silicate nanocomposites. Polymer Composites, 30, 343-350 (2009).

DOI: $10.1002 / p c .20591$

[29] Zhao D., Feng J., Huo Q., Melosh N., Fredrickson G. H., Chmelka B. F., Stucky G. D.: Triblock copolymer syntheses of mesoporous silica with periodic 50 to 300 angstrom pores. Science, 279, 548-552 (1998).

DOI: $\underline{10.1126 / \text { science } .279 .5350 .548}$
[30] Zhao D., Huo Q., Feng J., Chmelka B. F., Stucky G. D.: Nonionic triblock and star diblock copolymer and oligomeric surfactant syntheses of highly ordered, hydrothermally stable, mesoporous silica structures. Journal of the American Chemical Society, 120, 60246036 (1998). DOI: 10.1021/ja974025i

[31] Grenier-Loustalot M-F., Larroque S., Grenier P., Leca J-P., Bedel D.: Phenolic resins: 1. Mechanisms and kinetics of phenol and of the first polycondensates towards formaldehyde in solution. Polymer, 35, 30463054 (1994). DOI: 10.1016/0032-3861(94)90418-9

[32] Ji X., Hampsey E., Hu Q., He J., Yang Z., Lu Y.: Mesoporous silica-reinforced polymer nanocomposites. Chemistry of Materials, 15, 3656-3662 (2003).

DOI: $10.1021 / \mathrm{cm} 0300866$

[33] Hernández-Padrón G., Rojas F., García-Garduño M., Canseco M. A., Castaño V. M.: Development of hybrid materials consisting of $\mathrm{SiO}_{2}$ microparticles embedded in phenolic-formaldehydic resin polymer matrices. Materials Science and Engineering A, 355, 338-347 (2003).

DOI: 10.1016/S0921-5093(03)00101-1

[34] Hoffmann F., Cornelius M., Morell J., Fröba M.: Silica-based mesoporous organic-inorganic hybrid materials. Angewandte Chemie International Edition, 45, 3216-3251 (2006).

DOI: 10.1002 /anie.200503075

[35] Yu J., Huang X., Wang L., Peng P., Wu C., Wu X., Jiang P.: Preparation of hyperbranched aromatic polyamide grafted nanoparticles for thermal properties reinforcement of epoxy composites. Polymer Chemistry, 2, 1380-1388 (2011). DOI: $10.1039 / \mathrm{C} 1 \mathrm{PY} 00096 \mathrm{a}$ 